\title{
Soft Fault Detection and Localization in an Unshielded Twisted Pair Network using Power Line Communication
}

\author{
Abdel Karim Abdel Karim ${ }^{1,2} \mathbb{C}^{\mathrm{a}}$, Virginie Degardin ${ }^{2} \mathbb{C}^{\mathrm{b}}$, Vincent Cocquempot $^{1} \mathbb{C}^{\mathrm{c}}$ \\ and M. Amine Atoui ${ }^{1,2} \mathbb{O}^{\mathrm{d}}$ \\ ${ }^{1}$ Université de Lille, CNRS, Centrale Lille, UMR 9189 - CRIStAL, F-59000 Lille, France \\ ${ }^{2}$ Univ. Lille, CNRS, Centrale Lille, Univ. Polytechnique Hauts-de-France, UMR 8520 - IEMN, F-59000 Lille, France \\ \{abdel-karim.abdel-karim, virginie.degardin, vincent.cocquempot, matoui\}@univ-lille.fr
}

\begin{abstract}
Keywords: Vehicular Embedded Networks, Monitoring, Fault Detection, Fault Localization, Soft Fault, Transmission System.
\end{abstract}

\begin{abstract}
Vehicular electrical wires and communication systems can be affected by faults. Depending on their severity, faults can be divided into two families: hard and soft faults. Hard faults indicate open/short circuits that can lead to serious breakdowns because they prevent the flow of energy or information. Even though soft faults do not have such severe impacts on the system, they may develop into hard faults in the long term, hence the need to detect them. In this paper, an unshielded twisted pair cable that undergoes a water-tree degradation is considered. A soft fault, which may correspond to the effect of a mechanical constraint on the cable, is introduced as a series resistor. The studied network is a tree-shape network composed of one source and multiple receivers, one at each end of a branch. Assuming that these receivers operate in a healthy state, to detect the fault, the transfer function from each receiver are monitored and a detection index is used. Another index, based on the comparison of the effect of the fault at each endpoint, is proposed to locate the affected branch. To summarize the detection and localization algorithm, a signature matrix is generated. Simulation results are presented to illustrate our approach.
\end{abstract}

\section{INTRODUCTION}

As part of autonomous/electric vehicles, Advanced Driver Assistance Systems (ADAS) have been integrated into vehicles to ensure road safety and driving comfort (Lindgren and Chen, 2006). These systems rely on electronic equipments that exchange information via dedicated communication networks. Since the One-Pair Ether-Net Alliance has encouraged the adoption of the automotive Ethernet for modern vehicular networks, the studied network is based on Unshielded Twisted Pair (UTP) cables (Zweck and Communication, 2019). This network is subject to failures that can have fatal consequences. Hence the need of a monitoring system that can distinguish between healthy and faulty states. Reflectometry and its derivatives are the main used monitoring methods for fault detection in networks (Furse et al., 2020). Time

\footnotetext{
a (iD) https://orcid.org/0000-0003-4390-9858

b (D) https://orcid.org/0000-0002-8716-7940

c (1) https://orcid.org/0000-0001-7281-911X

d(D) https://orcid.org/0000-0003-1893-5819
}

Domain Reflectometry (TDR) and Frequency Domain Reflectometry (FDR) form the two main classes of reflectometry systems (Furse et al., 2003). The experiments using reflectometry realized by (Kafal and Benoit, 2018), on a two-wire transmission line, demonstrate that soft faults can have signatures which are much weaker than changes in impedance which can affect a cable during actual installation conditions. Furthermore, the measurement and simulations in (Griffiths et al., 2006), on a two-wire conductor General Cable SKU 02301.R5.02 lamp cord (Corporation, 2017), have also shown that these signatures are invisible using TDR, FDR and Spread-spectrum TDR in the frequency range of $100 \mathrm{MHz}-200 \mathrm{MHz}$ due to the normal impedance variation in the realistic environment of the cable. Moreover, even if the latter was detected, reflectometry based methods have a major drawback due to the use of special, expensive and bulky sensors and instruments (Huo et al., 2019). Finally, according to (Kafal and Benoit, 2018), the effect of nodes on a reflectogram can be much stronger than the effect of a soft fault. A Power Line Communication (PLC) system can also be used to monitor the 
network. In (Lallbeeharry et al., 2018), a PLC system implemented a real time fault detection between two modems. The time evolution of the transfer function $H(f)$ between the two ends of a UTP cable is monitored, and statistical coefficients (i.e. Correlation coefficient $\rho$ and Euclidean distance $E u$ ) are used to detect soft faults.

In this paper, a soft fault detection and localization method is proposed and analyzed in a tree-shape network composed of one source and multiple receivers, one at each end of a branch. The soft fault - which can appear, for example, after an electric discharge or may be due to a bad contact between a wire and a connector - is represented by the insertion of a series resistance such as in (Lallbeeharry et al., 2018), while the network is being degraded by a Water Tree (WT) phenomenon as described in (Huo et al., 2019). This phenomenon represents the change in the dielectric permittivity of a cable insulation caused by humidity. The transfer function from each point of view of the receivers, between the source and each receiver is measured by the receivers at the end of each branch of the network, through Orthogonal Frequency Division Multiplexing (OFDM) pilot symbols sending by the source. Afterwards, two indices extracted from $H(f)$ are used to detect the presence of a soft fault then to locate the affected branch. The detection index is calculated between a reference $H(f)$ and the successive ones which are measured. Once the presence of a fault is confirmed, a localization index is then computed to determine the branch where the fault is located. The fault detection and localization methodology proposed in this paper is general, but for presentation reason, a network comprising one source, three receivers and two nodes is studied. In section 2, the detection and localization indices is presented along with the proposed method. To validate this work, data are generated using cable, degradation and soft fault models that are detailed in section 3.1. Simulations and the results are carried on in section 3.2. Conclusions and future work to be conducted close this paper.

\section{FAULT DETECTION AND LOCALIZATION}

\subsection{Fault Diagnostic Indices}

The indices presented in this section are based only on the transfer function $H(f)$ of the network.

Definition 1. CORRELATION COEFFICIENT. The correlation coefficient $\rho_{i}$ expresses the distance be- tween the reference transfer function and the successive acquisitions (measurements) of the transfer function at each receiver $R_{i}$.

$$
\rho_{i}=\frac{\left|\sum_{k} H_{R_{i}}^{\text {Reference }}\left(f_{k}\right) \cdot H_{R_{i}}^{* \text { Test }}\left(f_{k}\right)\right|}{\sum_{k}\left|H_{R_{i}}^{\text {Reference }}\left(f_{k}\right)\right|^{2}}
$$

$H_{R_{i}}^{\text {Reference }}$ is the first acquisition that is supposed to be the transfer function for the healthy network at the receiver $R_{i} . H_{R_{i}}^{\text {Test }}$ is the measured transfer function that needs to be tested at a given time at the receiver $R_{i} . f_{k}$ denotes the frequency in the range of $1 \mathrm{MHz}$ to $100 \mathrm{MHz}$ with a step of $10 \mathrm{kHz}$. $X^{*}$ denotes the conjugate of $X$, and $|X|$ is the module of $X$.

Definition 2. DETECTION INDEX. The detection index $I_{D_{i}}$ is defined as :

$$
I_{D_{i}}=\left|1-\rho_{i}\right|
$$

In a healthy case, $I_{D_{i}}$ computed at each receiver $i$, is equal or close to zero and it deviates from zero when a fault occurs or in presence of network degradation. Once the fault is detected by one of the receiver, the network is considered as faulty.

Definition 3. LOCALIZATION INDEX. The localization index $I_{L_{i}}$ is defined as the ratio between transfer function measured after fault detection at each receiver $R_{i}$ and the transfer function measured before fault detection at each receiver $R_{i}$.

$$
I_{L_{i}}=\frac{H_{R_{i}}^{\text {Faulty }}(f)}{H_{R_{i}}^{\text {Healthy }}(f)}
$$

where $I_{L_{i}}$ denotes the localization index at the receiver $R_{i} . \quad H_{R_{i}}^{\text {Healthy }}(f)$ and $H_{R_{i}}^{\text {Faulty }}(f)$ are respectively the transfer function before and after the fault detection.

\subsection{Illustration on a Study Case}

To show the usefulness of the localization index, the three cases in Fig. 1 are explored. Let consider the multipath model found in (Zimmermann and Dostert, 1999), (Zimmermann and Dostert, 2002) that describes the propagation of the waves in a network. According to the multipath signal propagation model, there are several possible paths from the source $S$ to the receiver $R_{i}$. Furthermore, the signal is reflected at each impedance discontinuity (localized fault and node). The received signal is thus a superposition of the first $M$ significant paths considered. Hence, the transfer function $H(f)$ can be represented by the following equation :

$$
H(f)=\sum_{i=1}^{M} g_{i} \cdot e^{-\gamma \cdot d_{i}}
$$




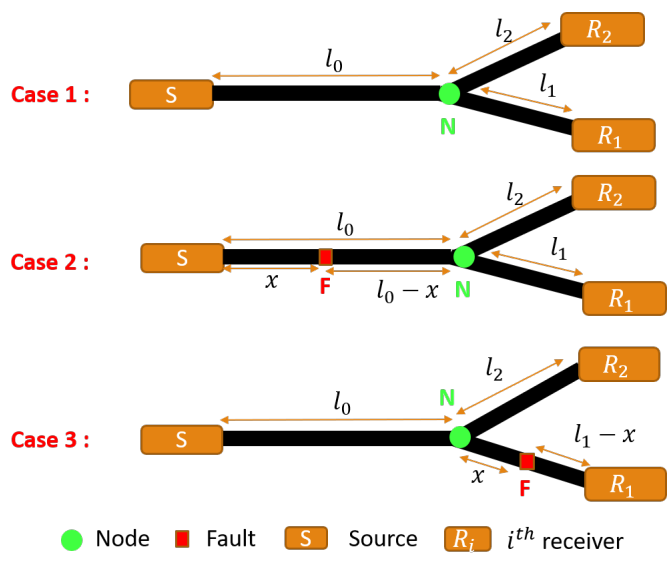

Figure 1: Y-Shaped networks.

where $M$ represents the number of the most significant paths, $g_{i}$ denotes the weighting factor of the path $i$ representing the product of the reflection and transmission factors along the path, $\gamma$ is the complex propagation constant and $d_{i}$ is the length of the path $i . \gamma$ and $g_{i}$ are both depending on the frequency $f$.

To simplify and to limit the number of paths $M$, the source and the receivers are considered matched to the network and the first two paths are taken into consideration.

\section{Healthy Network.}

In a healthy network, only the direct path between the source and each receiver exists. Thus, the transfer function from the point of view of each receiver is :

$$
\begin{aligned}
& H_{R_{1}}^{\text {Healthy }}(f)=t_{1 N} \cdot e^{-\gamma \cdot\left(l_{0}+l_{1}\right)} \\
& H_{R_{2}}^{\text {Healthy }}(f)=t_{1 N} \cdot e^{-\gamma \cdot\left(l_{0}+l_{2}\right)}
\end{aligned}
$$

where $t_{1 N}$ is the transmission factor of the node $\mathrm{N}, l_{0}, l_{1}$ and $l_{2}$ are the lengths of each branch presented in Fig. 1.

\section{Appearance of a Soft Abrupt Fault Before the} Node.

If a fault occurs at the branch situated before the node $\mathrm{N}$, several paths are possible.

From the point of view of $R_{1}$, the two most significant paths are :

- Path no. 1: S - F - N - $R_{1}$.

- Path no.2: $\mathrm{S}-\mathrm{F}-\mathrm{N}-\mathrm{F}-\mathrm{N}-R_{1}$.

Therefore, the frequency response from $\mathrm{S}$ to $R_{1}$ can be expressed as:

$$
\begin{aligned}
H_{R_{1}}(f) & =t_{F} \cdot t_{2 N} \cdot e^{-\gamma \cdot\left(l_{0}+l_{1}\right)}+ \\
& t_{F} \cdot r_{2 N} \cdot r_{F} \cdot e^{-\gamma \cdot 2 \cdot\left(l_{0}-x\right)} \cdot t_{2 N} \cdot e^{-\gamma \cdot\left(l_{0}+l_{1}\right)} \\
& =t_{F} \cdot\left(1+r_{2 N} \cdot r_{F} \cdot e^{-\gamma \cdot 2 \cdot\left(l_{0}-x\right)}\right) \cdot t_{2 N} \cdot e^{-\gamma \cdot\left(l_{0}+l_{1}\right)}
\end{aligned}
$$

By analogy for the second receiver $R_{2}$ :

$$
\begin{aligned}
H_{R_{2}}(f) & =t_{F} \cdot t_{2 N} \cdot e^{-\gamma \cdot\left(l_{0}+l_{2}\right)}+ \\
& t_{F} \cdot r_{2 N} \cdot r_{F} \cdot e^{-\gamma \cdot 2 \cdot\left(l_{0}-x\right)} \cdot t_{2 N} \cdot e^{-\gamma \cdot\left(l_{0}+l_{2}\right)} \\
& =t_{F} \cdot\left(1+r_{2 N} \cdot r_{F} \cdot e^{-\gamma \cdot 2 \cdot\left(l_{0}-x\right)}\right) \cdot t_{2 N} \cdot e^{-\gamma \cdot\left(l_{0}+l_{2}\right)}
\end{aligned}
$$

where $t_{F}$ and $r_{F}$ represent respectively the transmission and reflection factors of the fault. $t_{2 N}$ is the transmission factor of the node $N$. The attenuation due to the signal propagation from the source to each receiver $i, e^{-\gamma \cdot\left(l_{0}+l_{i}\right)}$, are multiplied by the same value in (7) and (8).

Hence, $I_{L_{1}}$ and $I_{L_{2}}$ are equal :

$$
I_{L_{1}}=I_{L_{2}}=t_{F} \cdot\left(1+r_{2 N} \cdot r_{F} \cdot e^{-\gamma \cdot 2 \cdot\left(l_{0}-x\right)}\right) \cdot \frac{t_{2 N}}{t_{1 N}}
$$

\section{Appearance of a Soft Abrupt Fault after the Node.}

If a fault occurs after the node $\mathrm{N}$ in the branch directly linked to the receiver $R_{1}$, several paths are also possible. From the point of view of $R_{1}$, the transfer function is expressed as:

$$
\begin{aligned}
H_{R_{1}}(f) & =t_{F} \cdot t_{3 N} \cdot e^{-\gamma \cdot\left(l_{0}+l_{1}\right)}+ \\
& t_{F} \cdot r_{3 N} \cdot r_{F} \cdot e^{-\gamma \cdot 2 \cdot x} \cdot t_{3 N} \cdot e^{-\gamma \cdot\left(l_{0}+l_{1}\right)} \\
& =t_{F} \cdot\left(1+r_{3 N} \cdot r_{F} \cdot e^{-\gamma \cdot 2 \cdot x}\right) t_{3 N} \cdot e^{-\gamma \cdot\left(l_{0}+l_{1}\right)}
\end{aligned}
$$

From the point of view of $R_{2}$, the transfer function is expressed as :

$$
\begin{aligned}
& H_{R_{2}}(f)=t_{3 N} \cdot e^{-\gamma \cdot\left(l_{0}+l_{2}\right)}+ \\
& \quad t_{3 N} \cdot r_{F} \cdot e^{-\gamma \cdot 2 \cdot x} \cdot t_{3 N} \cdot e^{-\gamma \cdot\left(l_{0}+l_{2}\right)} \\
& =\left(1+t_{3 N} \cdot r_{F} \cdot e^{-\gamma \cdot 2 \cdot x}\right) t_{3 N} \cdot e^{-\gamma \cdot\left(l_{0}+l_{2}\right)}
\end{aligned}
$$

where $r_{F}$ denotes the reflection coefficient of the fault. $t_{3 N}$ and $r_{3 N}$ are respectively the transmission and reflection coefficient of the node $\mathrm{N}$. In this case, the weighting factors from (10) and (11) are different. Therefore, $I_{L_{1}}$ is different than $I_{L_{2}}$ :

$$
\begin{aligned}
& I_{L_{1}}=t_{F} \cdot\left(1+r_{3 N} \cdot r_{F} \cdot e^{-\gamma \cdot 2 \cdot x}\right) \cdot \frac{t_{3 N}}{t_{1 N}} \\
& I_{L_{2}}=\left(1+t_{3 N} \cdot r_{F} \cdot e^{-\gamma \cdot 2 \cdot x}\right) \cdot \frac{t_{3 N}}{t_{1 N}}
\end{aligned}
$$

This index allows us to eliminate the effect of the attenuation due to the signal propagation from the source to the $i^{t h}$ receiver : $e^{-\gamma\left(l_{0}+l_{i}\right)}$. This index depends on the attenuation due to the propagation between the node and the fault positions : $e^{-\gamma \cdot 2 \cdot x}$, the severity of the fault represented by $r_{F}$ and/or $t_{F}$ and the impact of the fault on the node represented by the change of the transmission and the reflection factors of the node. 


\subsection{Fault Detection and Localization Method}

The proposed fault detection and localization method (see Fig. 2) is based on the two aforementioned indices.

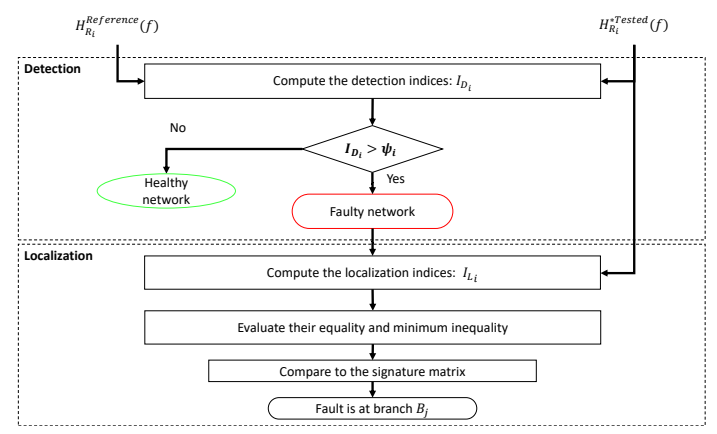

Figure 2: Fault detection and localization algorithm.

It is divided into two phases : "Detection" then "Localization". These phases are discussed in the following.

\section{Detection Phase:}

The network is considered as faulty, if, at least, one of the indices, $I_{D_{i}}$, computed at each receiver is higher than its predefined threshold $\psi_{i}$. Otherwise the network is considered as healthy. The threshold $\psi_{i}$ is empirically deduced to detect abrupt soft faults while taking into account the slow variation due to WT. The localization phase is triggered after the presence of a fault is confirmed.

\section{Localization Phase:}

When a fault is detected, the indices $I_{L_{i}}$ are computed. To determine the branch where the present fault is located, they are evaluated and compared to each branch's fault reference signature. This comparison would suggest a set of candidates. A candidate stands for the presence of fault in a branch $j$, denoted $F_{B_{j}}$. Multiple faults are out of the scope of this paper. Only single faults are assumed.

Table 1: Signature matrix.

\begin{tabular}{|c|c|c|c|}
\cline { 2 - 4 } \multicolumn{1}{c|}{} & $F_{B_{1}}$ & $\ldots$ & $F_{B_{N}}$ \\
\hline$I_{L_{1}}$ & $\alpha_{1,1}$ & $\cdots$ & $\alpha_{1, N}$ \\
\hline$\vdots$ & $\vdots$ & $\vdots$ & $\vdots$ \\
\hline$I_{L_{m}}$ & $\alpha_{m, 1}$ & $\ldots$ & $\alpha_{m, N}$ \\
\hline $\min _{R_{i}}\left(I_{L_{i}}\right)$ & $\beta_{1}$ & $\ldots$ & $\beta_{N}$ \\
\hline
\end{tabular}

The proposed signature matrix is derived and depends on the considered network's topology. The ma- trix rows correspond to the behaviour of $m+1$ indicators, the $m$ receiver's localization indices plus the set of receivers at which the localisation indices values are minimum, towards the presence of a fault $F_{B_{j}}$. Its columns describe the $N$ branch's fault reference signatures.

A general signature matrix is provided in table 1. It is characterized by two types of parameters: $\alpha$ and $\beta$. A parameters $\alpha_{i, j}$ represents a Boolean variable which takes its values in the set $\{0,1\}$. Equal localization indices relative to a fault in a branch $j$, admit a value 1 for their Boolean variables in the column $j$ of the signature matrix. The Boolean variables of those with different values take 0 . A parameter $\beta_{j}$ is a subset, in respect to $F_{B_{j}}$, of receivers at which the localisation indices' values are the lowest, such as $\beta_{j} \subset\left\{R_{1}, \ldots, R_{m}\right\}$.

The signature matrix is build in such a way that faults are decoupled in a set of tests such as the $m+1$ indicators reacts to a particular subset of faults and each branch's fault $j$ is characterised by a certain configuration of the indicators values. Different configurations arise depending on where the fault is located on the network. These configurations can be generalised by four cases.

- If a fault $F_{B_{j}}$ occurs prior to all the network's nodes, the closest branch to the source: all the $m$ indices computed at the receivers situated after it lead to a same value. The localization indices are equal, $\forall i, \alpha_{i, j}=1$.

- If a fault $F_{B_{j}}$ occurs behind all the network's nodes, at the end of the network: all the $m$ indices computed at the receivers situated before it take different values, $\forall i, k, \alpha_{i, j} \neq \alpha_{k, j}$. The localization index $i$ directly linked to the affected branch has the lowest value among all the indices, $\beta_{j}=I_{L_{i}}$.

- If a fault $F_{B_{j}}$ is located at the branch directly linked to a receiver $i$ : only the indices computed at the receivers situated after the nearest node to the receiver $i$ share the same values, for each $k, k>i, \alpha_{k, j}=1$. The index of localization related to the receiver $i$ has the lowest value among all the indices, $\beta_{j}=I_{L_{i}}$.

- If a fault $F_{B_{j}}$ occurs between two nodes, a branch not linked to any receiver: only the indices computed at the receivers situated before the farthest node, among the two nodes, to the source take different values, for each $k, k<i, \alpha_{i, j} \neq \alpha_{k, j}$. The receivers sharing the same values have the lowest values compared to the remaining indices' values. for each $k, k>i, I_{L_{k}} \in \beta_{j}$. 


\subsection{Summary}

The step-by-step procedure of the proposed fault detection and localization scheme based on PLC is summarized as follows:

1. Construct the signature matrix with respect to the network topology.

2. Define the reference $H(f)$ from each receiver's point of view.

3. Acquire successive $H(f)$ from each receiver $i$, compute and monitor $I_{D_{i}}$.

4. If no change is detected, return to step (3), otherwise go to the next step.

5. Compute the localization indices $I_{L_{i}}$ and evaluate their equality and minimum inequality.

6. Compare the resulting evaluation vector of step 5 to the fault signature in the signature matrix.

7. From step (6), deduce the faulty branch.

\section{APPLICATION CASE STUDY}

\subsection{Data Generation}

In this work, the studied network is the automotive Ethernet, which is based on Unshielded Twisted Pairs (UTP). This type of network is chosen for its ability to meet the needs of ADAS (Hank et al., 2013).

Simulations of such a network are used to validate the proposed method, before applying it in a real network. In this paper, a deterministic chain matrices -based model (Galli and Banwell, 2005) of the network is used to compute $H(f)$. The model is based on the physical characteristics of the network (topology, type of cable, terminal devices i.e. source and receivers) and on the transmission line theory. Each quadripole element of the network is represented by its corresponding ABCD chain matrix and the global ABCD chain matrix is obtained by the product of each individual element matrix (Peres et al., 2003). For UTP networks, chain matrices can be computed using the equations found in (Al-Asadi et al., 2000), (El Sahmarany, 2013), (Truong, 2000) and (Ramo et al., 1994). To our knowledge, the work by (El Sahmarany, 2013) is the only one between them that took the twisting phenomenon in consideration. Thus, the equations found in the latter one are used to compute those parameters in the marge of frequencies going from $1 \mathrm{MHz}$ to $100 \mathrm{MHz}$. These parameters are used in the deterministic model to deduce $H(f)$.

\subsubsection{Degradation and Soft Fault Model}

Degradation: The degradation is defined by an irreversible process in one or more of the primary parameters of the cable with either time, duration of use or an external cause (e.g. humidity, heat, etc ...). The WT degradation caused by humidity affects the dielectric permittivity of the wire $\varepsilon$. Since the capacitance of the cable is directly related to $\varepsilon$, then the WT degradation is represented as two capacitors as shown in Fig. 3 (Mugala, 2005).

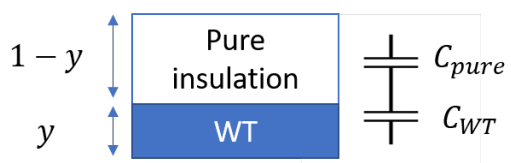

Figure 3: Representation of an aged sample.

A model of the WT degradation seen in (Förstel and Lampe, 2017) and (Sanniyati et al., 2016) is used to reproduce a degradation going from $0 \%$ to $5 \%$ with a step of $0.1 \%(y=0$ to $y=0.05)$. The total permittivity of an aged sample given by (Huo et al., 2019) is computed using (14) :

$$
\varepsilon_{\text {total }}=\frac{\varepsilon_{\text {insulator }} \varepsilon_{W T}}{y \varepsilon_{\text {insulator }}+(1-y) \varepsilon_{W T}}
$$

where $\varepsilon_{W T}$ is the permittivity of the affected zone shown in blue in Fig. 3. $\varepsilon_{\text {insulator }}$ is the permittivity of the pure insulation and $y$ is the percentage of affected zone.

In the following, the WT degradation is considered as a normal evolution of the cable characteristics.

Soft Fault: This type of faults only produces very small impedance changes (Auzanneau, 2013). Therefore, a soft fault is usually represented by a small localized modification of the characteristic impedance of the line. In this paper, the soft fault is represented by a sudden insertion of series resistor.

\subsection{Simulations and Results}

As an example, several simulations are conducted on the network shown in Fig. 4. This network has one source, three receivers, two nodes and five branches. Note that the source and all the receivers are matched to the line (Same impedance of the characteristic impedance of the network $Z_{c}=120 \Omega$ ).

The three transfer functions of the network are computed and their corresponding Bode diagram are shown in Fig. 5 and 6.

1. Detection: We assume that the level of degradation increases by $1 \%$ every 50 successive acquisi- 


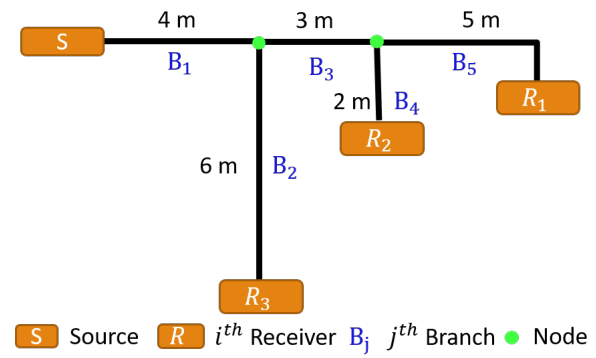

Figure 4: Unshielded twisted pair network.

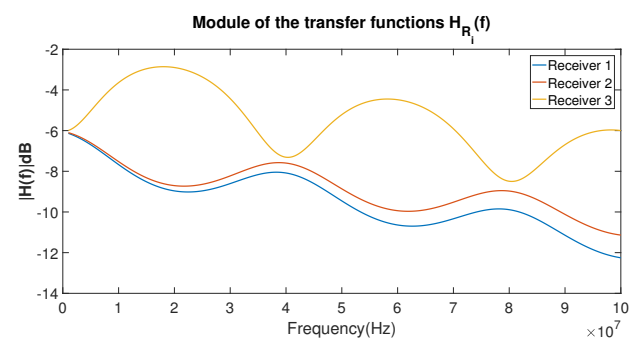

Figure 5: Module of the transfer function of each receiver $\left|H_{R_{i}}(f)\right|$.

tions of $H_{R_{i}}(f)$. In this case, where the cable undergoes a WT degradation with the absence of a soft fault, the computed $I_{D_{i}}$ between $H_{R_{i}}^{\text {reference }}(f)$ and $H_{R_{i}}^{\text {test }}(f)$ increases at a slow pace as shown in Fig. 7 for each receiver. $H_{R_{i}}^{\text {test }}(f)$ denotes the successive test data that needs to be tested at a given time. When an abrupt soft fault occurs, its effect differs from one receiver to another. A $5 \Omega$ resistor is inserted in series in the branch $B_{3}$ at $t=t_{0}$, a rupture in the variation of the detection index appears as shown in Fig. 8. Therefore, to detect the abrupt soft fault, it is sufficient to detect the rupture by one of the receiver.

2. Localization: Once the fault is detected, to locate the affected branch, the localization index $I_{L_{i}}$ is computed at each receiver. Since $I_{L_{i}}$ is a complex index, its module $\left|I_{L_{i}}\right|$ and phase $\phi\left(I_{L_{i}}\right)$ are presented in Fig. 9.

The results show that $I_{L_{1}}$ is equal to $I_{L_{2}}$ and both of them are different from $I_{L_{3}}$ as it has been proven

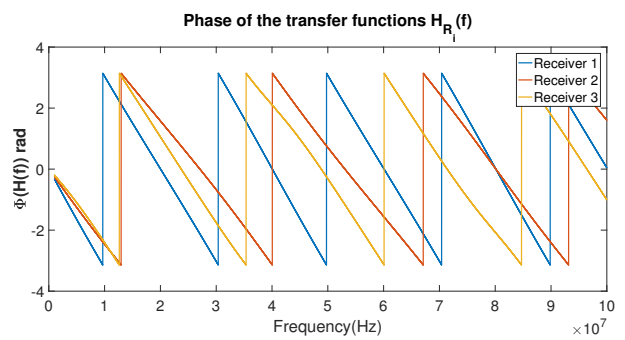

Figure 6: Phase of the transfer function of each receiver $\phi\left(H_{R_{i}}(f)\right)$.

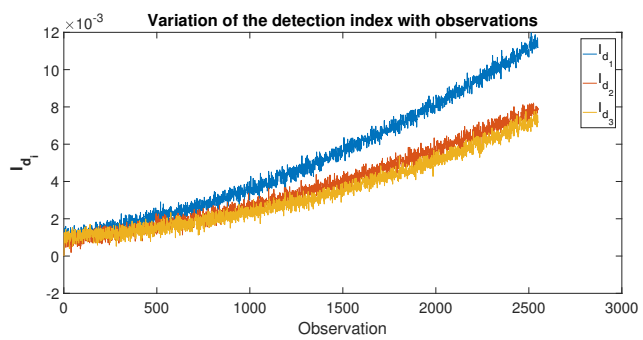

Figure 7: Variation of the detection indices (Degraded network without abrupt soft fault).

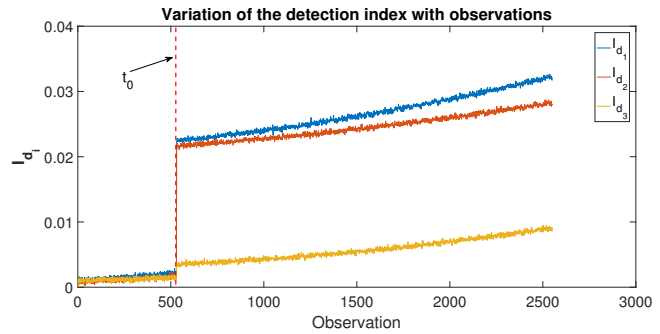

Figure 8: Variation of the detection indices (Degraded network with the appearance of a soft fault at $t_{0}$ ).

in section 2. By changing the position of the fault from one branch to another and observing the behavior of the localization indices a topology dependent signature matrix can be obtained.

Table 2: Signature matrix of the network in Fig.4.

\begin{tabular}{|c|c|c|c|c|c|}
\cline { 2 - 6 } \multicolumn{1}{c|}{} & $F_{B_{1}}$ & $F_{B_{2}}$ & $F_{B_{3}}$ & $F_{B_{4}}$ & $F_{B_{5}}$ \\
\hline$I_{L_{1}}$ & 1 & 1 & 1 & 0 & 0 \\
\hline$I_{L_{2}}$ & 1 & 1 & 1 & 0 & 0 \\
\hline$I_{L_{3}}$ & 1 & 0 & 0 & 0 & 0 \\
\hline $\min _{R_{i}}\left(I_{L_{i}}\right)$ & & $R_{3}$ & $R_{1} \cap R_{2}$ & $R_{2}$ & $R_{1}$ \\
\hline
\end{tabular}

Note that a 1 in the table represents equality and a 0 represents a difference. All the indices holding the number 1 are equal and the index holding the number 0 is different from all the other indices. For instance, let consider that the localized soft fault occurs at the branch $B_{2}$ or the branch $B_{3}$. The fault is then located before the node connected to the receivers $R_{1}$ and $R_{2}$, therefore $I_{L_{1}}$ is equal to
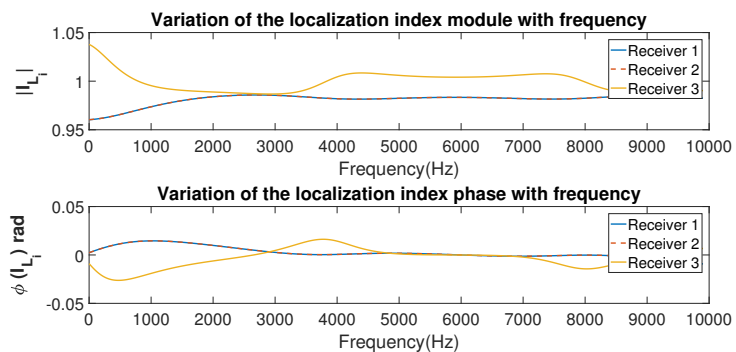

Figure 9: Variation of the localization index. A $5 \Omega$ resistor is inserted in series in the branch $B_{3}$ at $t=t_{0}$. 
$I_{L_{2}}$. To differentiate between these two cases (fault at $B_{2}$ or at $B_{3}$ ), the minimum index should then be computed. The branch $B_{2}$ is directly linked to $R_{3}$, thus if the fault is in this branch, the minimum index is $I_{L_{3}}$. If there was no exclusive minimum $\min \left(I_{L_{i}}\right)=I_{L_{1}}=I_{L_{2}}$, then the fault is in the common branch of the two receivers as in the case of fault in $B_{3}$.

\section{CONCLUSIONS AND FUTURE WORK}

In this paper, a fault detection and localization methodology based on the transfer function $H(f)$ is proposed to detect a soft fault. This methodology is based on two indices. At first, the detection index is computed. Once the fault is detected by one of the receivers, the localization index is computed at each endpoint of the network. The localization indices are checked against one another to verify whether they are equal or not. The results of these tests are compared to each branch fault's signature in the proposed signature matrix. The proposed matrix is constructed with respect to the network topology. To illustrate our proposal, two bus networks are considered: a degrading two nodes network and an Y-shaped (one node) network.

Further work will be realized to expand the validity and study the sensitivity of the detection and localization indices, in more complex networks. Also, it would be interesting to introduce statistical equality tests for better and accurate comparison between localization indices when noise is considered. This approach will be also validated on a test bench that is under construction.

\section{ACKNOWLEDGEMENTS}

This research work is done in the framework of the ELSAT2020 project which is co-financed by the European Union with the European Regional Development Fund, the French state and the Hauts-de-France Region Council.

\section{REFERENCES}

Al-Asadi, M. M., Duffy, A. P., Hodge, K. G., and Willis, A. J. (2000). Twisted pair cable design analysis and simulation. In 49th IWCS Symposium, pages 111-120.
Auzanneau, F. (2013). Progress In Electromagnetics Research B, Vol. 49, 253-279, 2013. Progress In Electromagnetics Research, 49(February):253-279.

Corporation, G. C. T. (2017). Cord \& Cordset Products for industrial, commercial and specialty applications.

El Sahmarany, L. (2013). Méthodes d'amélioration pour le diagnostic de câble par réflectométrie. $\mathrm{PhD}$ thesis, Université Blaise Pascal Clermont - Ferrand II.

Förstel, L. and Lampe, L. (2017). Grid diagnostics: Monitoring cable aging using power line transmission. In 2017 IEEE International Symposium on Power Line Communications and its Applications (ISPLC), pages 1-6. IEEE.

Furse, C., Chung, Y., Dangol, R., Nielsen, M., Mabey, G., and Woodward, R. (2003). Frequency-domain reflectometry for on-board testing of aging aircraft wiring. Electromagnetic Compatibility, IEEE Transactions on, 45:306 - 315 .

Furse, C., Kafal, M., Razzaghi, R., and Shin, y.-j. (2020). Fault diagnosis for electrical systems and power networks: A review. IEEE Sensors Journal, PP:1-1.

Galli, S. and Banwell, T. (2005). A novel approach to the modeling of the indoor power line channel-part ii: Transfer function and its properties. IEEE Transactions on Power Delivery, 20(3):1869-1878.

Griffiths, L., Parakh, R., Furse, C., and Baker, B. (2006). The invisible fray: A critical analysis of the use of reflectometry for fray location. Sensors Journal, IEEE, 6:697 - 706 .

Hank, P., Vermesan, O., Müller, S., and Van Den Keybus, J. (2013). Automotive ethernet: In-vehicle networking and smart mobility. Proceedings -Design, Automation and Test in Europe, DATE, pages 1735-1739.

Huo, Y., Prasad, G., Atanackovic, L., and Lampe, L. (2019). Cable diagnostics with power line modems for smart grid monitoring. IEEE Access, PP:1-1.

Kafal, M. and Benoit, J. (2018). Baselining: A critical approach used for soft fault detection in wire networks. International Journal of Digital Information and Wireless Communications (IJDIWC), 8:52-57.

Lallbeeharry, N., Mazari, R., Dégardin, V., and Trebosc, C. (2018). Plc applied to fault detection on in-vehicle power line. In 2018 IEEE International Symposium on Power Line Communications and its Applications (ISPLC), pages 1-5. IEEE.

Lindgren, A. and Chen, F. (2006). State of the art analysis: An overview of advanced driver assistance systems (adas) and possible human factors issues. $\mathrm{Hu}$ man Factors and Economic Aspects on Safety, pages 38-50.

Mugala, G. (2005). High frequency characteristics of medium voltage XLPE power cables. $\mathrm{PhD}$ thesis, KTH.

Peres, P., Souza, C., and Bonatti, I. (2003). Abcd matrix: A unique tool for linear two-wire transmission line modelling. International Journal of Electrical Engineering Education, 40.

Ramo, S., Whinnery, J. R., and Van Duzer, T. (1994). Fields and waves in communication electronics. John Wiley \& Sons. 
Sanniyati, C., Arief, Y., Adzis, Z., Muhamad, N., Ahmad, M., Abu, M., Sidik, M. A. B., and Lau, K. Y. (2016). Water tree in polymeric cables: A review. Malaysian Journal of Fundamental and Applied Sciences, 11:191-200.

Truong, T. K. (2000). Twisted-pair transmission-line distributed parameters. The Boeing $\mathrm{CO}$.

Zimmermann, M. and Dostert, K. (1999). A multi-path signal propagation model for the power line channel in the high frequency range. In Proc. IEEE Int. Symp. Power Line Commun. Appl, pages 45-51. Lancaster, UK.

Zimmermann, M. and Dostert, K. (2002). A multipath model for the powerline channel. IEEE Transactions on communications, 50(4):553-559.

Zweck, H. and Communication, E. A. (2019). 10 Mbps Ethernet Technology and the Challenges Facing Automotive Microcontrollers. 